\title{
Prevalence of COVID 19 Positive Cases Presenting to a Psychiatric Emergency Room
}

\author{
Jeffrey Cardenas ${ }^{1,2}$ ( Janine Roach ${ }^{1,2} \cdot$ Alex Kopelowicz $^{1,2}$ \\ Received: 31 August 2020 / Accepted: 12 March 2021 / Published online: 26 March 2021 \\ (c) The Author(s), under exclusive licence to Springer Science+Business Media, LLC, part of Springer Nature 2021
}

\begin{abstract}
To explore the prevalence of SARS-CoV2 infection in the psychiatric emergency room setting. A Cross-sectional retrospective chart review was used to determine the point-prevalence of SARS-CoV2 infection and the characteristics of those infected. Of the patients tested for SARS-CoV2, 23/1057 (2.2\%) were positive. Most of these patients were homeless (living on the street) or came from congregate living settings. The high percentage of SARS-CoV2 positive psychiatric patients coming from congregate living settings stresses the importance of asymptomatic screening in this vulnerable population.
\end{abstract}

Keywords SARS-CoV2 - COVID-19 · Psychiatric emergency room · Psychiatric patients · Congregate setting $\cdot$ Point prevalence

\section{Introduction}

On 12/31/2019 the Wuhan Municipal Health Commission in China reported a cluster of pneumonia cases in Wuhan caused by a novel coronavirus that was eventually identified as SARS-CoV2, causing a disease called COVID 19 (World Health Organization, 2020). The virus rapidly spread to multiple countries with the first confirmed case of 2019$\mathrm{nCoV}$ infection reported in the United States on January 20, 2020 (Holshue et al. 2020). The pandemic has posed particular challenges for populations in congregate settings, such as psychiatric patients admitted to hospitals or living in residential facilities. In February 2020, approximately 50 inpatients and 30 mental health professionals in a psychiatric hospital in Wuhan, Hubei province, China were diagnosed with COVID-19 (China News Weekly, 2020). Psychiatric facilities have had to design new processes and

Jeffrey Cardenas

jcardenas@dhs.lacounty.gov

Janine Roach

jroach@dhs.lacounty.gov

Alex Kopelowicz

akopelowicz@dhs.lacounty.gov

1 Olive View UCLA Medical Center, 14445 Olive View Dr. Cottage H1, Sylmar, CA, USA

2 Department of Psychiatry \& Biobehavioral Sciences, UCLA David Geffen School of Medicine, Los Angeles, CA, USA protocols to deliver care in a manner that mitigates the risk of transmission among patients and staff. A study by Bojdani et al. reviewed the impact the pandemic has had on psychiatric services, including psychiatric emergency rooms. They noted that psychiatric emergency rooms have had to develop screening protocols, deliver care via the use of various technologies, and develop testing protocols. (Bojdani et al., 2020) The present study aims to delineate the extent to which psychiatric patients presenting to a psychiatric emergency room were positive for COVID-19. We also provide a qualitative description of those patients who tested positive for COVID-19.

\section{Methods}

The hospital's IRB reviewed the study protocol and determined it to be exempt. The study followed a cross-sectional retrospective design to calculate the point prevalence of COVID-19 among subjects admitted to the Psychiatric Emergency Room (PER) between 4/20/20 and 7/20/20 at Olive View-UCLA Medical Center- a safety net hospital in Los Angeles County. A retrospective chart review was performed for all patients presenting to the PER. The PER patient log was used to compile a list of all patients presenting to the PER during that time period. Patient identifiers were removed and all data was de-identified. Only patients admitted to the PER were tested for COVID-19. 
As such, patients who self-presented to the Psych ER as triages but were not admitted were not tested for COVID-19 and are excluded from the analysis. Psychiatric patients with COVID-19 admitted to the medical unit from the medical ER were not included because they did not originate in the PER. Two patients admitted to the PER refused testing and were also excluded from the analysis. Of note, some patients were discharged and later readmitted during this time period. Their tests were counted as distinct results as there was a possibility the patient could have been re-infected during their time in the community.

Upon admission to the PER, a patient's vital signs were taken and patients were assessed for the presence of cough, fever, shortness of breath, and diarrhea. Further, they were screened to determine if they were arriving from a congregate living arrangement. Symptomatic patients and those arriving from a congregate setting were immediately placed in isolation. For both symptomatic and asymptomatic patients, a nasopharyngeal sample was collected by two RNs wearing masks, face shields, and gowns to test for the presence of SARS-CoV-2 (COVID-19). Asymptomatic patients with pending SARS-CoV2 tests were placed in a negative pressure room with other patients who were awaiting test results. Patients were encouraged to remain 6 feet apart and wear surgical masks. Symptomatic patients or patients arriving from congregate settings were kept in individual isolation rooms until their tests resulted. Patients were introduced into the general PER population once they received a negative test result. Masking and distancing were also encouraged in these areas. Patients who tested positive were maintained in isolation rooms.

At the time of the study, Olive View-UCLA Medical Center was utilizing tests from the following companies: Quest Diagnostics, Cepheid Gene Xpert, Cobas, and CDC SARS-CoV2 assay. The electronic medical record was queried for patient's SARS-CoV2 results as well as demographic data: age, race, gender, and most recent housing status (including a congregate setting). In addition, data was collected on COVID-19 test result, urine drug screen, presenting medical symptoms, co-morbidities and psychiatric diagnosis. Point prevalence was calculated by dividing the number of subjects who tested positive for COVID-19 by the total number of subjects admitted to the PER who received a COVID-19 test during the time period denoted above.

\section{Results}

There were 1174 total patient encounters at the PER between $4 / 20 / 20$ and $7 / 20 / 20$. One hundred seventeen of those patients were not tested; 115 were not admitted to the PER and 2 refused the test. SARS-CoV-2 testing was completed for 1057 patients.
Of the patients included in the analysis, 23/1057 (2.2\%) patients tested positive for SARS-CoV-2 (Table 1). Of these 23 patients, 7/23 (30\%) were female. The age range of the patients was 13-66; median age of the positive patients was 34 years. Fourteen of the $23(61 \%)$ patients were Latinx, 4/23 (17\%) were Black and 4/23 (17\%) were white. For comparison, $27.4 \%$ of the patients admitted to the PER in the same time period were Latinx and $11.4 \%$ were Black. Nine of twenty three patients (39\%) were homeless and living on the street, $6 / 23(26 \%)$ were housed in a non-congregate setting, and 8/23 (35\%) came from congregate living situations such as jail (4/23), substance use rehabilitation facility $(2 / 23)$, skilled nursing facility $(1 / 23)$ and board and care facility (1/23). Fifty-two percent of the patients $(12 / 23)$ tested positive for an illicit substance on urine toxicology screen. Of the twenty-three COVID 19 positive patients, $13 / 23(57 \%)$ were diagnosed with a psychotic disorder on discharge, 7/23 (30\%) were diagnosed with an affective disorder, and 10/23 (43\%) were diagnosed with a substance use disorder. Of note, some patients received more than one diagnosis.

\section{Discussion}

\section{Main Findings}

The prevalence rate of the Olive-View Medical Center's PER population during this time period $(2.2 \%)$ was about $50 \%$ higher than the prevalence in Los Angeles County at the endpoint of our study (1.5\%) (Los Angeles County Department of Public Health, 2020).

Of the 23 COVID-19 positive patients in our study, 8/23 (35\%) arrived from congregate living settings, which is almost five times higher than the percentage of COVID-19 positive patients $(6.9 \%)$ in congregate living settings seen in LA County (Los Angeles County Department of Public Health, 2020). It must be noted, however, that the figure for the percentage of COVID-19 positive patients in congregate living settings may be an underestimate as testing in these settings has been limited by the shortage of available testing in LA County at the time.

Asymptomatic SARS-CoV-2 detection in congregate settings is especially concerning in patients with serious mental illness as they are more likely to have pre-morbid conditions such as diabetes, COPD, and heart failure: all risk factors for a poor prognosis with SARS-CoV-2 infection. (Crump et al. 2013; Parohan et al. 2019; Woodhead et al. 2014). Patients with serious mental illness are known to have deficits in working memory (Rose \& Ebmeier, 2006; Mathias et al., 2018). A recent study shows that these deficits may interfere with adherence to distancing protocols, thus putting this 
Table 1 Demographics, housing status, drug screen results, psychiatric diagnoses, medical comorbidities, and COVID 19 related symptoms of patients found to be COVID-19 positive in the psychiatric emergency room

\begin{tabular}{|c|c|c|c|c|c|c|c|c|c|}
\hline $\mathrm{Pt}$ & Date & Gender & Race & Age & Housing & Urine tox screen & Diagnosis & Comorbidity & Medical Symptom \\
\hline 1 & $4 / 24 / 20$ & M & B & 25 & Homeless & Neg & Psychosis & None & None \\
\hline 2 & $4 / 28 / 20$ & $\mathrm{~F}$ & $\mathrm{~L}$ & 51 & Jail & $\mathrm{Neg}$ & Mood & None & None \\
\hline 3 & $4 / 30 / 20$ & M & $\mathrm{L}$ & 21 & Jail & $\mathrm{Neg}$ & Psychosis & None & None \\
\hline 4 & $5 / 3 / 20$ & $\mathrm{~F}$ & B & 46 & SNF & Amphetamine & Psychosis/ substance & DM/HTN & Cough \\
\hline 5 & $5 / 4 / 20$ & $\mathrm{~F}$ & $\mathrm{~L}$ & 34 & Housed & Barbiturates & Mood & None & None \\
\hline 6 & $5 / 11 / 20$ & M & $\mathrm{L}$ & 44 & Housed & Neg & Substance & Morbid obesity & None \\
\hline 7 & $5 / 15 / 20$ & M & $\mathrm{L}$ & 31 & Rehab & $\mathrm{Neg}$ & Mood/substance & None & None \\
\hline 8 & $5 / 22 / 20$ & M & $\mathrm{W}$ & 32 & Rehab & Amphetamine/cannabis & Mood/substance & None & Anosmia \\
\hline 9 & $5 / 23 / 20$ & $\mathrm{~F}$ & W & 39 & Homeless & Neg & Psychosis & None & None \\
\hline 10 & $5 / 26 / 20$ & M & $\mathrm{L}$ & 13 & Housed & Cannabis & Mood & None & None \\
\hline 11 & $6 / 9 / 20$ & M & $\mathrm{L}$ & 41 & Homeless & Amphetamine/cannabis/ cocaine & Psychosis/substance & None & None \\
\hline 12 & $6 / 13 / 20$ & M & $\mathrm{L}$ & 26 & Housed & Neg & Mood & None & None \\
\hline 13 & $6 / 19 / 20$ & M & $\mathrm{L}$ & 28 & Homeless & Amphetamine & Psychosis/substance & None & None \\
\hline 14 & $6 / 19 / 20$ & M & $\mathrm{L}$ & 33 & Housed & Neg & Mood & None & Cough \\
\hline 15 & $6 / 23 / 20$ & M & $\mathrm{L}$ & 28 & Jail & Amphetamine/cannabis & Psychosis & None & None \\
\hline 16 & $6 / 25 / 20$ & M & B & 34 & Homeless & Amphetamine/cannabis & Substance & Ulcerative colitis & None \\
\hline 17 & $6 / 28 / 20$ & $\mathrm{~F}$ & $\mathrm{O}$ & 26 & Homeless & Neg & Substance & None & None \\
\hline 18 & $7 / 2 / 20$ & M & B & 66 & Jail & Amphetamine/cannabis & Psychosis & DM/HTN & None \\
\hline 19 & $7 / 4 / 20$ & $\mathrm{~F}$ & $\mathrm{~W}$ & 35 & Homeless & Cannabis & Psychosis/substance & None & None \\
\hline 20 & $7 / 7 / 20$ & M & $\mathrm{L}$ & 25 & Homeless & Amphetamines & Psychosis/substance & None & Cough \\
\hline 21 & $7 / 9 / 20$ & M & W & 55 & $\mathrm{~B} \& \mathrm{C}$ & Neg & Psychosis/substance & None & None \\
\hline 22 & $7 / 12 / 20$ & $\mathrm{~F}$ & $\mathrm{~L}$ & 25 & Homeless & Amphetamines/cannabis & Psychosis & None & None \\
\hline 23 & $7 / 16 / 20$ & M & $\mathrm{L}$ & 34 & Housed & $\mathrm{Neg}$ & Psychosis/substance & Asthma & None \\
\hline
\end{tabular}

$M$ male, $F$ female, $L$ Latinx, $B$ Black, $W$ white, $O$ other, $S N F$ skilled nursing facility, $B \& C$ board and care facility, $D M$ diabetes, $H T N$ Hypertension

population at higher risk of infection and spreading the virus (Xie et al. 2020).

In the Olive View-UCLA Medical Center's psychiatric emergency room, we attempted to prevent a COVID-19 outbreak within the psychiatric units of the hospital by testing all patients admitted. All but four of the 23 COVID-19 positive patients were asymptomatic on the day of testing. SARS-CoV-2 detection is critically important for infection control in congregate settings, specifically psychiatric emergency rooms and inpatient psychiatric units. Our PER has rooms which can hold up to 10 patients and 5 staff at any given time, so we were concerned about possible infection and rapid contagion in these settings. As of the time of the submission of this manuscript (July 20,2020), there has not been an outbreak in the hospital in which the index case originated in the psychiatric emergency room.

\section{Strengths}

We are unaware of research involving the point-prevalence of SARS-CoV-2 infection in asymptomatic psychiatric patients. We were able to test 1055 of 1057 patients admitted to our PER regardless of symptom status or prior exposure, which gives us information on the prevalence of infection in our patient sample without test selection bias.

\section{Limitations}

This was a retrospective, cross-sectional study focused on clinical characteristics using a simple statistical analysis; there was not a comparator group of non-psychiatric patients. Another limitation of the study was the reliance on the self-report of symptoms; patients with serious mental illness are less likely to recognize their own medical conditions and self-report may be inaccurate (Kilbourne et al. 2006). Data was based on a SARS-CoV-2 test on admission, which may not detect infections early on in the illness course. A number of different SARS-CoV-2 PCR NP swab tests were used, and the sensitivity and specificity of each of these tests are not entirely understood at this time. Some of the obstacles we faced in implementing our new processes and protocols included having limited space to house the different categories of patients, the length of time it took to get results, and initially the limited amount of testing. Although 
we only had two patients refuse testing, this can also pose an obstacle. Some strategies that can prove to be useful include explaining to patients that refusing to be tested will result in having to remain isolated from the general milieu, reattempting to persuade patients throughout their stay as their psychiatric symptoms mitigated, and offering oropharyngeal testing.

\section{Conclusion}

These data demonstrate the point-prevalence of SARSCoV-2 during a three-month period in patients presenting to a psychiatric emergency room at a public hospital in Los Angeles early in the pandemic. The finding of asymptomatic infections in these patients highlights the importance of detecting infection in a population that is more likely to have underlying health conditions and is less likely to present for medical care. Thus far, the protocol of immediate assessment of symptoms, testing of all patients introduced into congregate settings, and daily monitoring of symptoms appears to have prevented an outbreak of COVID-19 within the psychiatric units of the hospital. However, it will be necessary to continue to monitor the rate of psychiatric patients who test positive for COVID-19 as the pandemic continues and potentially test and trace high risk populations.

Funding Alex Kopelowicz receives funding from the National Institute of Nursing Research. Other two authors have no financial support to disclose.

Data Availability Available.

\section{Declarations}

Conflict of interest The authors declare that they have no conflict of interest.

Ethical Approval Given Exempt Status by hospital IRB.

\section{References}

Bojdani, E., Rajagopalan, A., Chen, A., et al. (2020). COVID-19 pandemic: Impact on psychiatric care in the United States. Psychiatric Research., 289, 113069.
China News Weekly. (2020). Hospital-acquired infection in Wuhan Mental Health Center: around 80 medical staff and patients were diagnosed with 2019-nCoV pneumonia (in Chinese). Retrieved February 9, 2020, from https://news.sina.com.cn/c/2020-02-08/ doc-iimxxste9892538.shtml.

Crump, C., Sundquist, K., Winkleby, M. A., Sundquist, J., et al. (2013). Comorbidities and mortality in bipolar disorder: A Swedish national cohort study. JAMA Psychiatry, 70, 931-939.

Holshue, M. L., DeBolt, C., Lindquist, S., et al. (2020). First case of 2019 novel coronavirus in the United States. New England Journal of Medicine, 382, 929-936.

Kilbourne, A. M., McCarthy, J. F., Welsh, D., \& Blow, F. (2006). Recognition of co-occurring medical conditions among patients with serious mental illness. The Journal of Nervous and Mental Disease, 194, 598-602.

Los Angeles County Department of Public Health. (2020). LA County COVID-19 survelliance dashboard. Retrieved July 21, 2020, from http://dashboard.publichealth.lacounty.gov/covid19_surveillance_ dashboard.

Los Angeles County Department of Public Health. (2020). Los Angeles County residential congregrate and acute care settings with an active COVID-19 outbreak including at least one laboratoryconfirmed resident. Retrieved July 21, 2020, from http://publi chealth.lacounty.gov/media/coronavirus/locations.htm\#resid ential-settings.

Mathias, S. R., Knowles, J. B., Barrett, J., et al. (2018). Deficits in visual working-memory capacity and general cognition in African Americans with psychosis. Schizophrenia Research, 193, 100-106.

Parohan, M., Yaghoubi, S., Seraji, A., et al. (2020). Risk factors for mortality in patients with Coronavirus disease 2019 (COVID-19) infection: A systematic review and meta-analysis of observational studies. The Aging Male. https://doi.org/10.1080/13685538.2020. 1774748.

Rose, E. J., \& Ebmeier, K. P. (2006). Pattern of impaired working memory during major depression. Journal of Affective Disorders, 90, 149-161.

Woodhead, C., Ashworth, M., Schofield, P., \& Henderson, M. (2014). Patterns of physical co-/multi-morbidity among patients with serious mental illness: A London borough-based cross-sectional study. BMC Family Practice, 15, 117-126.

World Health Organization. (2020). Coronavirus disease 2019 (COVID-19). Retrieved July 21, 2020, from https://www.who.int/ news-room/detail/27-04-2020-who-timeline---covid-19.

Xie, W., Campbell, S., \& Zhang, W. (2020). Working memory capacity predicts individual differences in social-distancing compliance during the COVID-19 pandemic in the United States. PNAS, 2020(117), 17667-17674.

Publisher's Note Springer Nature remains neutral with regard to jurisdictional claims in published maps and institutional affiliations. 